\title{
EchoGéo
}

27 | 2014

Structures et armatures urbaines

\section{Les petites villes, de nouveaux centres pour le développement territorial chinois}

L'exemple de la province du Zhejiang

\section{Stéphane Milhaud}

\section{(2) OpenEdition}

Journals

\section{Édition électronique}

URL : https://journals.openedition.org/echogeo/13730

DOI : 10.4000/echogeo.13730

ISSN : 1963-1197

Éditeur

Pôle de recherche pour l'organisation et la diffusion de l'information géographique (CNRS UMR 8586)

\section{Référence électronique}

Stéphane Milhaud, «Les petites villes, de nouveaux centres pour le développement territorial chinois », EchoGéo [En ligne], 27 | 2014, mis en ligne le 20 mars 2014, consulté le 10 août 2021. URL : http:// journals.openedition.org/echogeo/13730 ; DOI : https://doi.org/10.4000/echogeo.13730

Ce document a été généré automatiquement le 10 août 2021.

EchoGéo est mis à disposition selon les termes de la licence Creative Commons Attribution - Pas d'Utilisation Commerciale - Pas de Modification 4.0 International (CC BY-NC-ND) 


\title{
Les petites villes, de nouveaux centres pour le développement territorial chinois
}

\author{
L'exemple de la province du Zhejiang
}

\author{
Stéphane Milhaud
}

\section{Armature urbaine et développement régional en Chine}

1 Depuis trente ans en Chine, l'urbanisation se poursuit à un rythme soutenu. La progression du taux d'urbanisation est exceptionnelle : de $17,9 \%$ en 1978, soit alors moins de la moitié du taux d'urbanisation de la planète, à $49,9 \%$ en $2010^{1}$, date à laquelle il rejoint sensiblement la moyenne mondiale. La moitié de la population chinoise est aujourd'hui urbaine. Sur cette période, elle a pratiquement quadruplé, passant de 172 à 670 millions $^{2}$, ce qui représente annuellement environ 16 millions de nouveaux citadins, un accroissement qui devrait encore perdurer pendant une à deux décades ${ }^{3}$ : c'est l'équivalent, chaque année, d'une nouvelle ville comme Shanghai.

2 L'exode massif des populations rurales du centre et de l'Ouest vers les villes du littoral a été déclenché par l'ouverture économique du pays lancée par Deng Xiaoping en 1978, date charnière à laquelle se sont engagées une croissance économique et une urbanisation uniques à une telle échelle. La Chine hérite alors d'une armature urbaine d'abord marquée par la formation de villes-régions au cours de la période impériale ${ }^{4}$, puis, à partir de 1842 et la signature du traité de Nankin, conclusion de la première guerre de l'Opium, elle est surtout façonnée par les concessions étrangères (Sanjuan, 2000, p. 19-22). Celles-ci sont à l'origine du développement de grandes villes (Shanghai, Wuhan, Tianjin), dont l'économie fleurit suite au commerce et aux échanges avec les pays étrangers. En 1949, à la proclamation de la République populaire de Chine, le gouvernement engage une politique de développement des petites et moyennes villes, puis les villes connaissent deux décennies de recul suite à l'échec du Grand Bond en avant et à des politiques davantage orientées vers les campagnes (Chan, 1994a, 
p. 24-27). Ainsi en 1978 la Chine est-elle encore majoritairement rurale, avec un taux d'urbanisation inférieur à $20 \%$.

3 À la fin $\mathrm{du} \mathrm{XX}^{\mathrm{e}}$ siècle, après plus d'une décennie d'une ouverture économique commencée sur le littoral puis progressivement diffusée vers les terres de l'intérieur, le développement régional prend la forme de bandes longitudinales, du littoral, vers le centre et l'Ouest, en cohérence avec le degré et le type de développement économique. Depuis, les géographes ont tour à tour adapté ce schéma aux évolutions en cours, soulignant la prééminence des trois espaces métropolitains du littoral autour de Pékin, de Shanghai et de Canton et réhabilitant une armature urbaine à l'échelle du pays en équilibre avec la métropolisation du littoral et son aire de rayonnement (Sanjuan, 2007, p. 157-185).

4 Cette armature urbaine souligne l'émergence de larges agglomérations, dont le développement est soutenu par les investissements des institutions internationales ${ }^{5}$, qui parlent de geographic cluster ou de regional urban system. Au début des années 1990, Terry McGee a modélisé la formation de ces espaces-régions, dénommés desakota (McGee, 1991), qui sont caractérisés par de fortes densités rurales, et organisés autour de centres urbains de différentes tailles où les activités non agricoles ne sont pas concentrées uniquement dans un cercle d'influence périurbain, mais également le long des axes de communication. En 2009, une recherche publiée par l'OCDE (Kamal-Chaoui, Leman,Rufei, 2009, p. 26-30) mettait ainsi en évidence 28 espaces-régions de ce type, appelés systèmes urbains régionaux ${ }^{6}$. Le territoire chinois se lirait ainsi au travers de la formation de ces régions urbaines composées de villes et de bourgs de toutes tailles et caractérisées par la densité des réseaux de communication, par une urbanisation en marche, et par la concentration des activités économiques et de la population ( $c f$. illustration 1).

5 L'armature urbaine ne se limite pas pour autant à ce découpage. Ainsi une récente étude du McKinsey Global Institute (McKinsey, 2009) souligne-t-elle que les tendances actuelles soutiennent le scénario de la croissance d'un grand nombre de villes de tailles moyennes disséminées sur le territoire ${ }^{7}$. Ce scénario rejoint la volonté du gouvernement, qui souhaite contenir la pression démographique exercée sur les grandes métropoles du pays au premier rang desquelles Pékin, Shanghai et Canton, tout en favorisant une meilleure répartition de la croissance économique. D'autre part, à l'intérieur des régions urbaines, la question de la place et du rayonnement des centres de différentes tailles reste en suspens. Ces espaces sont-ils entièrement structurés autour d'une métropole dominante, ou le résultat plus complexe d'un jeu d'échelles entre les bourgs, les villes de différentes tailles et la métropole? 


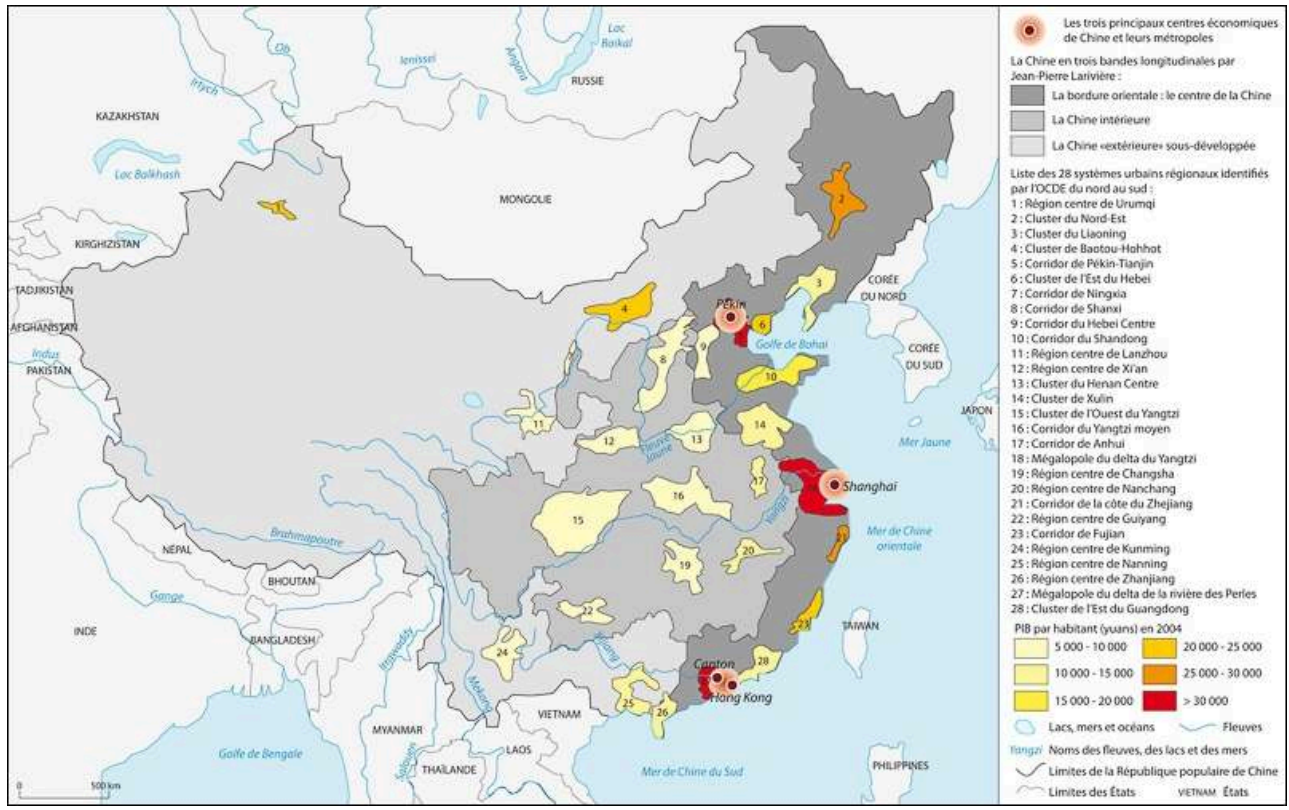

Sources : pour les données de 2004 : Kamal-Chaoui L., Leman E., Rufei Z., 2009. Urban Trends and Policy in China, p. 28 ; pour les données de 2010 : Zhongguo tongji nianjian [Annuaire statistique de la Chine], 2011, p. 44, 57 et 95 .

Ce sont les questions auxquelles nous tentons d'apporter un nouvel éclairage en prenant le cas particulier de la province du Zhejiang. Voisine de Shanghai sur le littoral, son taux d'urbanisation est passé de $13,7 \%$ en 1980 à $61,6 \%$ en 2010 , soit douze points au-dessus de la moyenne nationale. En 2010, la province a une population de 54,5 millions d'habitants et, avec un PIB par habitant de 50867 yuans, elle fait partie des territoires les plus riches de Chine, juste derrière les municipalités de Shanghai, Pékin et Tianjin et la province du Jiangsu ${ }^{8}$. Le Zhejiang recoupe en son Nord la région du delta du Yangzi (région métropolitaine de Shanghai), un des trois bassins industriels et démographiques les plus importants de Chine, avec le corridor Pékin-Tianjin (région métropolitaine de Pékin) et le delta de la rivière des Perles (région métropolitaine de Canton). Ainsi le Zhejiang permet-il d'étudier l'évolution de l'armature urbaine à la fois au sein d'une région urbaine majeure de l'espace chinois ainsi qu'au sein d'espaces dits périphériques, économiquement en retrait et situés plus à l'intérieur des terres. L'illustration 2 décrit la situation de cette province, qui peut être divisée en trois grands ensembles : la région deltaïque, le littoral et les terres intérieures.

- La région deltaïque du Nord-Est : elle recouvre le sud du delta du Yangzi et les rives de la baie de Hangzhou. Situées dans l'arrière-pays de Shanghai, ce sont des basses terres humides aux fortes densités humaines, $1040 \mathrm{hab} / \mathrm{km}^{2}$, dont la forme du développement s'apparente à un desakota. La région deltaïque recouvre le quart du Zhejiang, regroupe environ la moitié de sa population avec 24 millions d'habitants et compte pour $67 \%$ de son PIB'.

- Le littoral: il est composé d'une étroite bande continentale, entre mer et montagne. Il rassemble $29 \%$ de la population provinciale et $20 \%$ de son $\mathrm{PIB}^{10}$, et s'affirme comme le deuxième pôle de développement de la province. Si la densité brute du littoral, $810 \mathrm{hab} / \mathrm{km}^{2}$, est légèrement inférieure à celle de la région deltaïque, cela est dû à la zone montagneuse sous-peuplée qui le compose. L'étroite bande habitable est en fait bien plus dense que la 
région deltaïque. Le manque d'espace et la très forte densité démographique sont les caractéristiques principales de cet ensemble.

- Les terres intérieures : elles regroupent $21 \%$ de la population provinciale et concentre $13 \%$ de son $\mathrm{PIB}^{11}$, et constituent l'ensemble le plus en retard de la province. Les terres intérieures comportent des chaînes collinaires, des chaînes de montagnes et deux bassins fluviaux, le bassin de la rivière Qiantang et le bassin du Jinqu. Avec $248 \mathrm{hab} / \mathrm{km}^{2}$, elles sont en moyenne bien moins denses que la région deltaïque ou le littoral. La plus forte densité atteint $454 \mathrm{hab} / \mathrm{km}^{2}$ dans la plaine alluviale du bassin du Jinqu ${ }^{12}$.

Illustration 2 - La province du Zhejiang et ses territoires

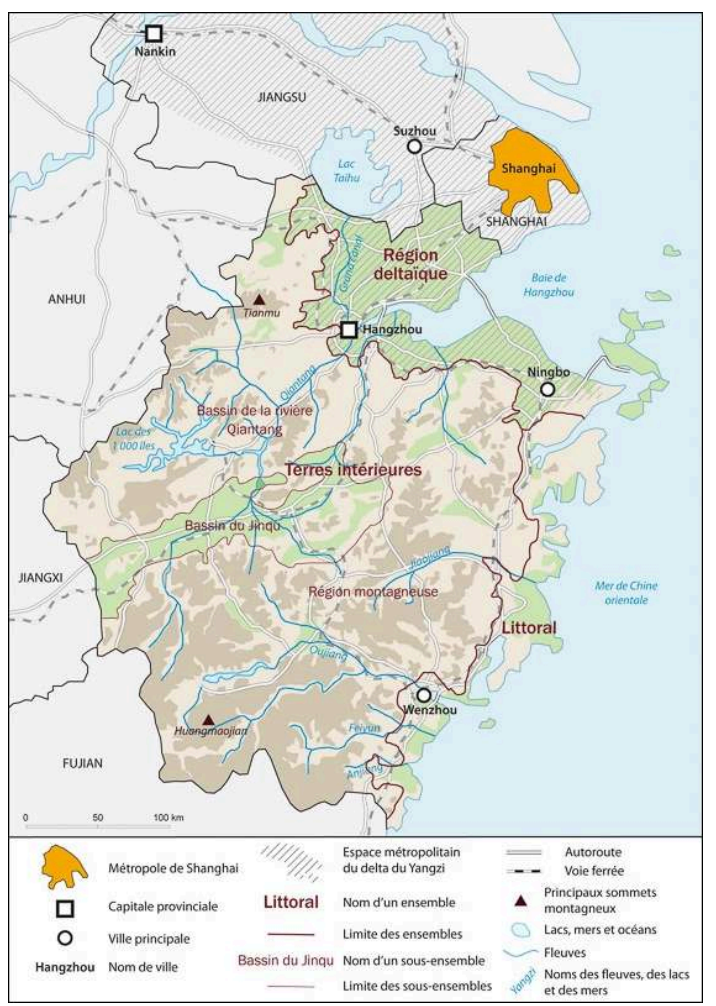

Source : Google Earth, 2012

7 Avant d'en analyser les évolutions démographiques et économiques, il convient de clarifier la définition de l'armature urbaine que nous utiliserons. La notion de ville se révèle en effet complexe en Chine, ayant une signification administrative différente de sa signification physique: la ville "administrative" ne s'arrête pas aux limites urbaines.

\section{L'intégration de la ville dans le système territorial}

Dans la langue chinoise, deux termes sont utilisés en référence à la notion de ville : shi 市et chengshi 城市. L'entité physique correspond au terme chinois chengshi, qui renvoie aux deux principaux rôles anciens de la cité : la défense (cheng 城, le rempart) et le commerce (shi 市, le marché) ${ }^{13}$. Il révèle que la ville était le centre politique et commercial du territoire en dépit d'une population majoritairement rurale. En revanche le caractère shi 市 utilisé sans cheng城 désigne le statut administratif d'un territoire, et signifie dans ce cas-là que le dit territoire a le statut de «ville », que l'on 
traduira alors par municipalité. Cette entité territoriale n'est pas caractérisée par un paysage uniquement urbain, mais elle regroupe des espaces à la fois urbains et ruraux, à l'intérieur d'un territoire excédant largement la taille d'une ville et davantage comparable à la taille d'un département français ${ }^{14}$. Ainsi les limites administratives d'une municipalité ne dépendent-elles nullement d'une continuité du bâti ou des réseaux. Dès lors, deux types d'entités administratives cohabitent dans l'espace chinois : les entités traditionnelles (province, préfecture et district) et les entités ayant le statut de municipalité. Les municipalités ont un rang administratif calqué sur les échelons traditionnels de la hiérarchie administrative. Il existe par conséquent trois types de municipalités: les municipalités de rang provincial (shengji shi 省级市), les municipalités de rang préfectoral (diji shi 地级市) et les municipalités de rang de district (xianji shi 县级市).

L'illustration 3 synthétise l'architecture territoriale pour la province du Zhejiang. Cinq niveaux administratifs se succèdent : celui de la province, de la préfecture, du district, du canton et du village. Au niveau préfectoral, les préfectures ont désormais disparu au profit des municipalités de rang préfectoral. Au niveau du district se trouvent les districts, les municipalités de rang de district, et les arrondissements. Au niveau du canton se trouvent les quartiers (jiedao 街道), les bourgs (zhen 镇) et les cantons (xiang 乡). Les arrondissements et les municipalités de rang de district sont composés de quartiers, de bourgs et de cantons, eux-mêmes composés de comités de résidents et de comités de villageois. Enfin les districts sont composés de bourgs et de cantons, euxmêmes également composés de comités de résidents et de comités de villageois.

Illustration 3 - La structure de l'administration territoriale dans la province du Zhejiang

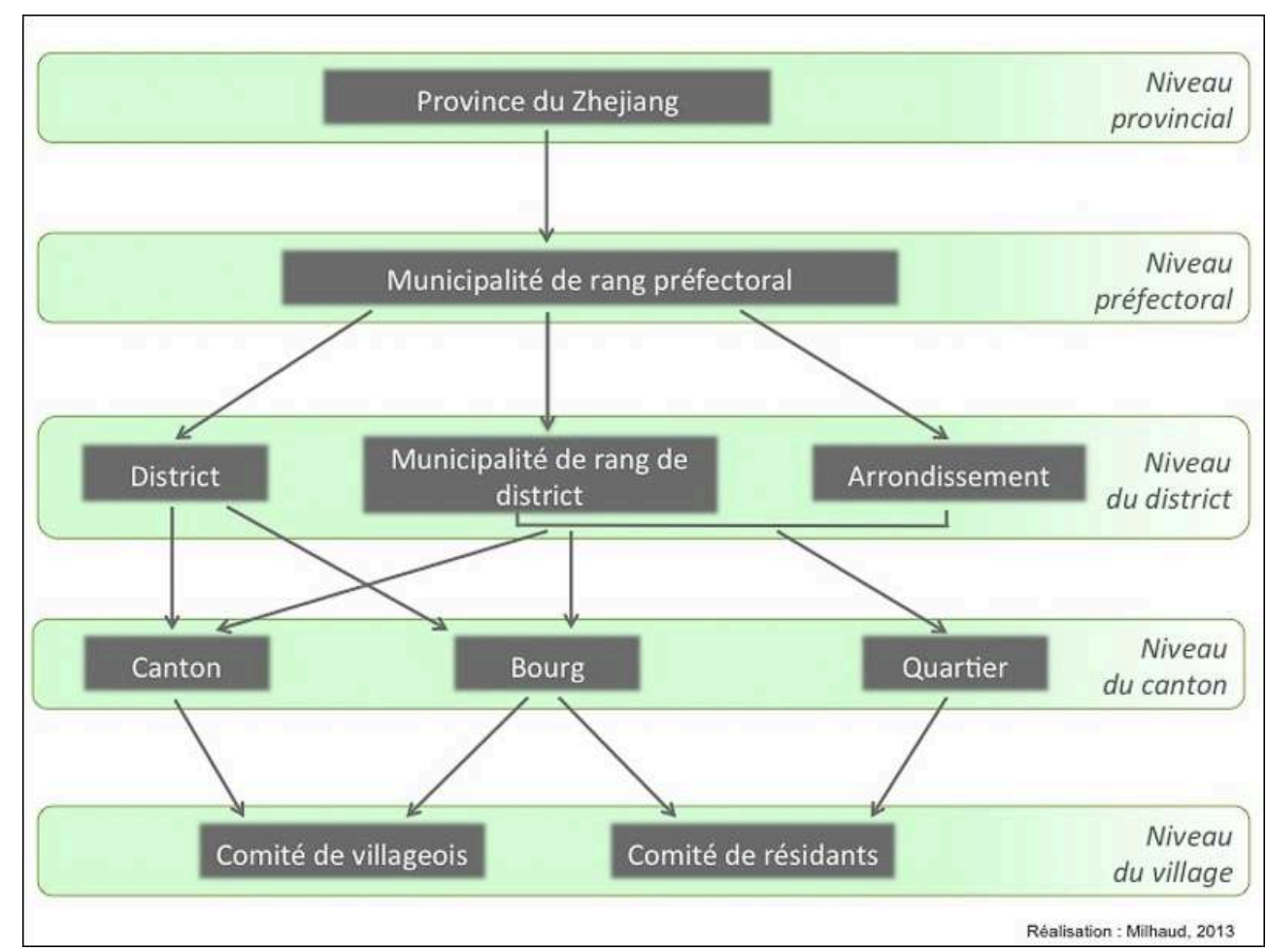

Auteur : S. Milhaud, 2013

Le découpage administratif du territoire n'épouse donc pas les limites des entités urbaines et rurales: une municipalité ou même un arrondissement n'est pas un 
territoire uniquement urbain, un district regroupe également une mixité d'espaces urbains et d'espaces ruraux. L'échelle administrative diffère de l'échelle urbaine.

Nous définirons ici la ville par son rôle de centre administratif du système territorial. L'architecture administrative définit la municipalité et le district. La ville est le centre administratif de la municipalité ou du district (chengshi). Le district n'ayant pas le statut de municipalité, nous appellerons son centre administratif non pas ville, mais bourg. Ainsi dénommerons-nous le centre administratif ville-centre lorsqu'il s'agit d'une municipalité et bourg-centre lorsqu'il s'agit d'un district.

L'illustration 4 propose une représentation spatiale de l'encadrement administratif du territoire et y identifie la ville, entité physique, qui retrouve son sens traditionnel de centre administratif du territoire (chengshi). La structure administrative esquisse donc en premier lieu une armature territoriale faite de municipalités et de districts, et l'armature urbaine apparaît à l'intérieur de cette armature territoriale: elle est constituée des centres administratifs de cette armature territoriale.

Illustration 4 - L'implantation territoriale de la ville chinoise

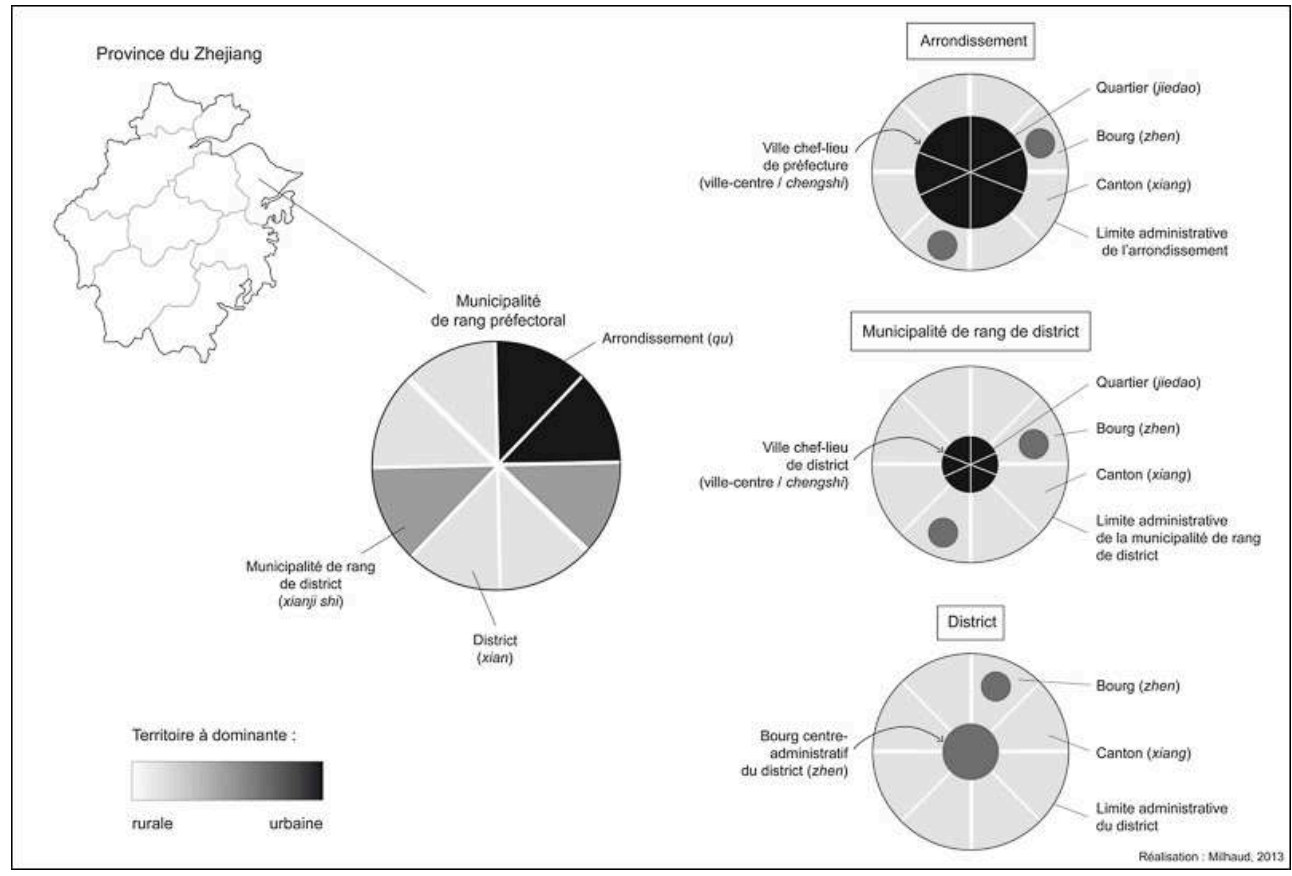

Auteur: S. Milhaud, 2013

13 Le schéma administratif territorial traduit la vision qu'ont les Chinois de la ville, considérée au sein d'un ensemble plus vaste que le périmètre urbain. Cet ensemble trouve sa justification dans les interactions entre espaces urbains et ruraux : la ville est vue comme le noyau d'un territoire qu'elle domine et qu'elle structure, l'un et l'autre étant liés par des activités complémentaires. Les espaces ruraux ont autant besoin des fonctions délivrées par la ville-centre que cette dernière se nourrit des ressources fournies par les campagnes. En ne séparant pas administrativement les espaces urbains des espaces ruraux, les synergies en sont facilitées.

À l'échelle de la province du Zhejiang, nous avons opté pour trois catégories de villes la grande ville, la ville moyenne et la petite ville -, dont les seuils démographiques nous ont paru les plus adaptés pour en analyser l'armature urbaine. Ils ont été choisis en 
considérant l'évolution de la taille des villes dans la province au cours des vingt dernières années, dans le but de constituer des catégories homogènes. La grande ville est une ville-centre d'une municipalité de rang préfectoral, qui a une population supérieure à un million d'habitants. La ville moyenne peut être une ville-centre d'une municipalité de rang préfectoral ou de rang de district. Sa population est comprise entre 500000 et 1 million d'habitants. Enfin la petite ville peut être une ville-centre d'une municipalité de rang de district ou un bourg centre-administratif de district. Sa population est comprise entre 100000 et 500000 habitants. Le tableau 1 synthétise les seuils de population caractérisant la petite ville, la ville moyenne et la grande ville au sein de l'armature urbaine. En 2010, quatre grandes villes se sont détachées avec une population supérieure à un million d'habitants. Ensuite, cinq villes ont aujourd'hui une population comprise entre 500000 et 1 million d'habitants, et 48 villes ont une population comprise entre 100000 et 500000 habitants.

Tableau 1 - Les critères de définition de l'armature urbaine

\begin{tabular}{|c|c|c|c|}
\hline $\begin{array}{l}\text { Armature } \\
\text { urbaine }\end{array}$ & $\begin{array}{l}\text { Territoire } \\
\text { administratif }\end{array}$ & Réalité morphologique & Population (habitants) \\
\hline Grande ville & $\begin{array}{l}\text { Municipalité de rang } \\
\text { préfectoral }\end{array}$ & $\begin{array}{l}\text { Ville-centre administratif } \\
\text { de préfecture }\end{array}$ & $\begin{array}{l}\text { Population ville-centre > } \\
1 \text { million }\end{array}$ \\
\hline \multirow{2}{*}{$\begin{array}{l}\text { Ville } \\
\text { moyenne }\end{array}$} & $\begin{array}{l}\text { Municipalité de rang } \\
\text { préfectoral }\end{array}$ & $\begin{array}{l}\text { Ville-centre administratif } \\
\text { de préfecture }\end{array}$ & \multirow{2}{*}{$\begin{array}{l}500000<\text { Population ville- } \\
\text { centre }<1 \text { million }\end{array}$} \\
\hline & $\begin{array}{l}\text { Municipalité de rang de } \\
\text { district }\end{array}$ & $\begin{array}{l}\text { Ville-centre administratif } \\
\text { de district }\end{array}$ & \\
\hline \multirow{2}{*}{ Petite ville } & $\begin{array}{l}\text { Municipalité de rang de } \\
\text { district }\end{array}$ & $\begin{array}{l}\text { Ville-centre administratif } \\
\text { de district }\end{array}$ & $\begin{array}{l}100000<\text { Population ville- } \\
\text { centre }<500000\end{array}$ \\
\hline & District & $\begin{array}{l}\text { Bourg-centre administratif } \\
\text { de district }\end{array}$ & $\begin{array}{l}100000<\text { Population bourg- } \\
\text { centre }<500000\end{array}$ \\
\hline
\end{tabular}

\section{Le développement des petites villes dans le Zhejiang}

À l'intérieur des trois grands ensembles de la province, pourtant fort différents, le développement des petites villes se révèle être une constante de l'évolution de l'armature urbaine au cours des deux dernières décennies. Les chiffres ci-après se réfèrent aux recensements de la population réalisés dans le Zhejiang en 1990, 2000 et $2010^{15}$.

\section{L'émergence des petites villes entre 1990 et 2000}

En 2000, l'armature urbaine se compose comme suit: quatre grandes villes - Hangzhou, Ningbo, Wenzhou et Taizhou -, une ville moyenne - Shaoxing - et 45 petites villes. Entre 1990 et 2000, le tableau 2 indique le taux de croissance annuel moyen des personnes possédant un hukou ${ }^{16}$ non agricole, c'est-à-dire de la population dont 
l'activité est officiellement enregistrée comme urbaine, suivant les catégories de ville : ce sont les petites villes qui connaissent les croissances les plus élevées, 4,09\% en croissance annuelle moyenne, bien au-dessus des grandes villes et des villes moyenne, respectivement 2,35 et 2,95\%. D'autre part, si l'on compare les grands ensembles, ce phénomène se manifeste sur le littoral avec un taux d'accroissement de la population non agricole de $4,53 \%$, près de deux fois celui de la région deltaïque, qui reste stable à $2,76 \%$.

Tableau 2 - Les petites villes, lieux des plus fortes croissances des hukou non agricoles entre 1990 et 2000 dans la province du Zhejiang

\begin{tabular}{|l|l|l|l|}
\hline Armature urbaine & $\begin{array}{l}\text { hukou non agricole } \\
\text { en } 1990\end{array}$ & $\begin{array}{l}\text { hukou non agricole } \\
\text { en } 2000\end{array}$ & $\begin{array}{l}\text { Taux d'accroissement } \\
\text { annuel moyen }\end{array}$ \\
\hline Grandes villes & 2704795 & 3411200 & $2,35 \%$ \\
\hline Ville moyenne & 184825 & 247100 & $2,95 \%$ \\
\hline Petites villes & 3499037 & 5224100 & $4,09 \%$ \\
\hline Ensemble & $\begin{array}{l}\text { hukou non agricole } \\
\text { en } 1990\end{array}$ & $\begin{array}{l}\text { hukou non agricole } \\
\text { en } 2000\end{array}$ & $\begin{array}{l}\text { Taux } \\
\text { annuel moyen }\end{array}$ \\
\hline Région deltaïque & 3802719 & 4992100 & $2,76 \%$ \\
\hline Littoral & 1351202 & 2104900 & $4,53 \%$ \\
\hline Terres intérieures & 1234736 & 1785400 & $3,76 \%$ \\
\hline $\begin{array}{l}\text { Bassin de la rivière } \\
\text { Qiantang }\end{array}$ & 240918 & 354500 & $3,94 \%$ \\
\hline Bassin du Jinqu & 714335 & 1013700 & $4,09 \%$ \\
\hline Montagnes du sud & 279483 & 200 & $36 \%$ \\
\hline
\end{tabular}

Sources : Zhejiang sheng 1990 nian renkou pucha ziliao [Résultats du recensement de la population de la province du Zhejiang en 1990], 1992, vol. 1, p. 32-43 ; Zhejiang sheng 2000 nian renkou pucha ziliao [Résultats du recensement de la population de la province du Zhejiang en 2000], 2002, vol. 1, p. 2-7.

17 L'illustration 5 donne la répartition dans le Zhejiang de ces taux de croissance entre 1990 et 2000 : les villes dont les taux de croissance du hukou non agricole sont les plus élevés, c'est-à-dire supérieurs à $5 \%$, sont exclusivement des petites villes, alors que les trois plus grandes villes de la province restent dans la tranche la plus basse, inférieure à $2,8 \%$. De plus, sur les 12 petites villes les plus dynamiques, huit sont situées sur le littoral. 
Illustration 5 - L'armature urbaine dans le Zhejiang en 2000 et son évolution entre 1990 et 2000

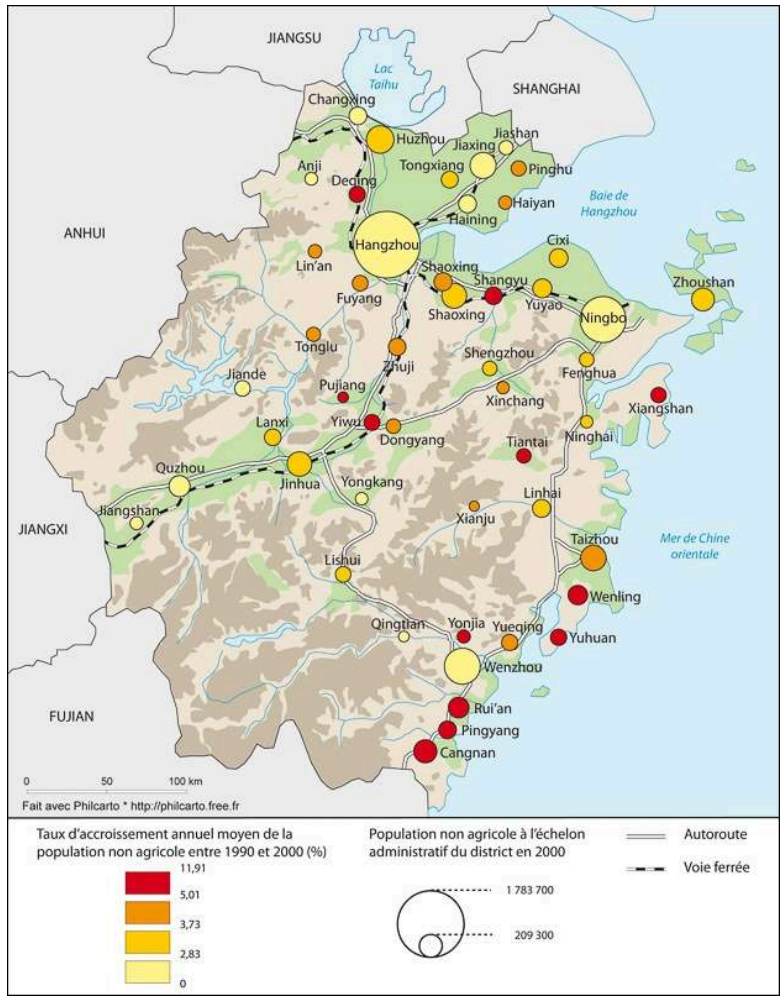

Sources : Zhejiang sheng 1990 nian renkou pucha ziliao, p. 2-6 ; Zhejiang sheng 2000 nian renkou pucha ziliao, p. 11-16.

De 1990 à 2000, la croissance urbaine se manifeste donc en priorité dans les petites villes et sur le littoral de la province : les chiffres du hukou montrent que les petites villes équilibrent peu à peu l'armature urbaine. Alors qu'en 1990 se dégageaient Hangzhou, Ningbo, Wenzhou et quelques centres secondaires ${ }^{17}$, l'architecture régionale du maillage urbain évolue : objets d'un processus dynamique d'urbanisation, les petites villes s'intègrent dans la hiérarchie et composent désormais un pôle d'équilibre en pleine expansion. En 2000, elles regroupent une population urbaine de 12832177 habitants, contre 633118 pour l'unique ville moyenne et 8405227 pour les grandes villes ${ }^{18}$.

\section{La confirmation de la croissance des petites villes entre 2000 et 2010}

19 Au cours de la décennie suivante, entre 2000 et 2010, le nombre des grandes villes reste stable, avec Hangzhou, Wenzhou, Ningbo et Taizhou, les quatre principaux centres de la province. En revanche celui des villes moyennes augmente: cinq villes de la catégorie des petites villes en 2000 ont désormais une population supérieure à 500000 habitants et passent dans la catégorie des villes moyennes. Il s'agit de Rui'an, Yiwu, Jiaxing, Huzhou et Jinhua. Ce constat confirme la croissance constante des petites entités urbaines, les faisant de facto changer de statut. Quant aux petites villes, leur nombre augmente de 45 en 2000 à 48 en 2010. Si l'on considère que cinq d'entre elles sont passées entre 2000 et 2010 dans la catégorie supérieure et qu'une ville moyenne 
(Shaoxing) rétrograde au statut de petite ville, cela équivaut à l'apparition de sept nouvelles petites villes sur cette période.

Le tableau 3 décrit l'évolution de l'armature urbaine entre 2000 et 2010 par catégories et ensembles. Bien que la croissance des grandes villes soit élevée, à 3,29\% de moyenne annuelle, les petites villes présentent un taux de croissance encore supérieur : 4,25\%. En ce qui concerne la répartition de la croissance urbaine, la région deltaïque et le littoral maintiennent des taux de croissance importants, respectivement 4,05 et 3,74\% par an. Mais cette décennie se caractérise surtout par la croissance urbaine dans les territoires de l'intérieur: le bassin de la rivière Qiantang, le bassin du Jinqu et les montagnes du sud affichent des taux plus élevés, respectivement 5,27, 4,61 et 4,83\%, signes d'un glissement des dynamiques urbaines.

Tableau 3 - L'urbanisation de l'intérieur du Zhejiang et la croissance continue des petites villes entre 2000 et 2010

\begin{tabular}{|l|l|l|l|}
\hline Armature urbaine & $\begin{array}{l}\text { Population urbaine } \\
\text { en } 2000\end{array}$ & $\begin{array}{l}\text { Population urbaine } \\
\text { en } 2010\end{array}$ & $\begin{array}{l}\text { Taux d'accroissement } \\
\text { annuel moyen }\end{array}$ \\
\hline Grandes villes & 8405227 & 11621267 & $3,29 \%$ \\
\hline Ville moyenne & 633118 & 643199 & $0,16 \%$ \\
\hline Petites villes & 12832177 & 19461880 & $4,25 \%$ \\
\hline Ensemble & $\begin{array}{l}\text { Population urbaine } \\
\text { en } 2000\end{array}$ & Population urbaine & $\begin{array}{l}\text { Taux } \\
\text { en } 2010\end{array}$ \\
\hline Région deltaïque & 10625267 & 15800402 & $4,05 \%$ \\
\hline Littoral & 6670596 & 9628358 & $3,74 \%$ \\
\hline Terres intérieures & 4574659 & 7288841 & $4,77 \%$ \\
\hline $\begin{array}{l}\text { Bassin de la rivière } \\
\text { Qiantang }\end{array}$ & 724091 & 1210557 & $5,27 \%$ \\
\hline $\begin{array}{l}\text { Bassin du Jinqu } \\
\text { Montagnes du sud }\end{array}$ & 1014948 & 4451506 & $4,61 \%$ \\
\hline
\end{tabular}

Sources : Zhejiang sheng 2000 nian renkou pucha ziliao, vol. 1, p. 2-7 ; Zhejiang sheng 2010 nian renkou pucha ziliao, vol. 1, p. 2-7.

21 L'illustration 6 présente la répartition de cette croissance urbaine entre 2000 et 2010 : les petites villes poursuivent leur expansion entamée dix ans plus tôt. Elles affichent les taux de croissance les plus hauts, c'est-à-dire supérieurs à 5,85\%. Les plus dynamiques d'entre elles se trouvent en priorité dans la région deltaïque (Changxing, Jiashan, Pinghu, Shaoxing, Yuyao, Daishan) et dans le bassin du Jinqu (Lin'an, Pujiang, Quzhou, Wuyi, Jinyun). Le littoral apparaît légèrement en retrait, seul Rui'an atteignant un taux de croissance comparable. 


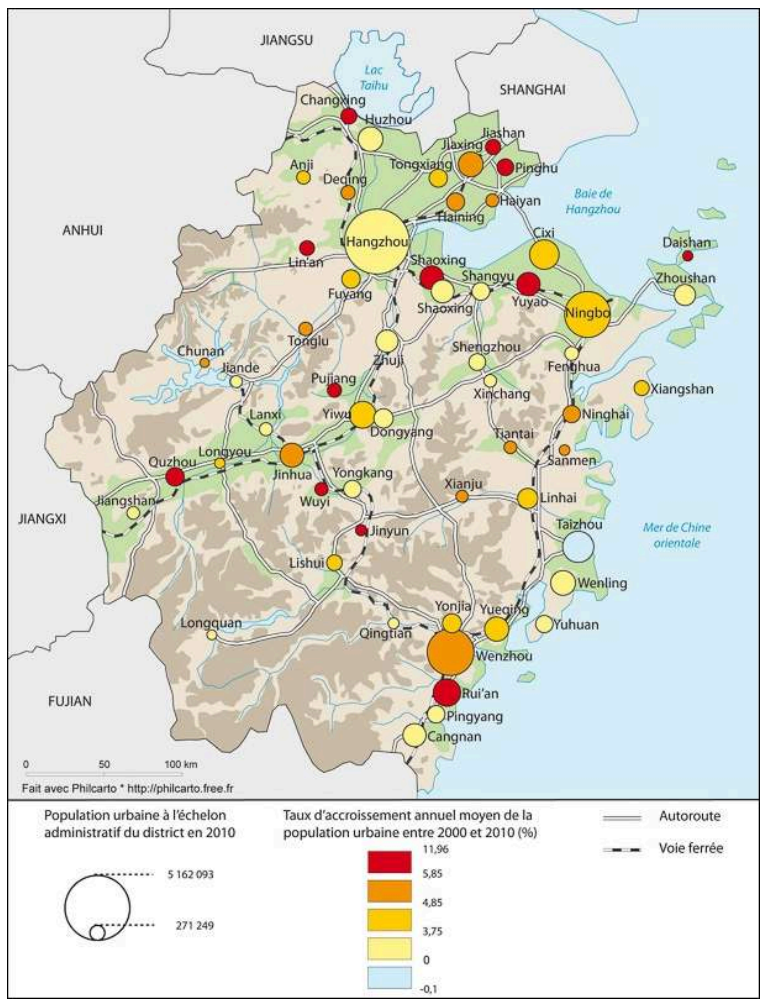

Sources : Zhejiang sheng 2000 nian renkou pucha ziliao, p. 2-6; Zhejiang sheng 2010 nian renkou pucha ziliao, vol. 1, p. 2-6.

Ainsi l'apparition de ce nouvel échelon semble-t-il équilibrer une armature urbaine ne reposant plus uniquement sur les trois ou quatre grands pôles et se répartissant désormais sur l'ensemble du territoire. Avec des taux de croissance démographique supérieurs à ceux des grandes villes, les petites villes s'affirment comme de nouveaux lieux de l'urbanisation dans le Zhejiang.

\section{La décentralisation et l'essor des gouvernements locaux}

L'émergence des petites villes correspond à leur intégration et au renforcement de leurs compétences au sein d'un système administratif territorial atypique, processus qui en a fait des pôles de gestion de territoires bien plus vastes que leur strict périmètre urbain et regroupant campagnes, villages et bourgs.

Dès le lancement des réformes économiques en 1978, la libéralisation du marché s'est accompagnée d'une décentralisation des pouvoirs au profit des gouvernements des municipalités et des districts, qui sont promus acteurs clés de l'urbanisation ${ }^{19}:$ dès lors, leurs centres administratifs vont pleinement bénéficier de cette décentralisation, qui leur attribue la gestion du développement économique et de l'aménagement du territoire. La volonté du gouvernement est de confirmer les villes en tant que moteurs de la croissance, et d'en faire des centres d'organisation et de gestion des activités aussi bien urbaines que rurales ${ }^{20}$. Dotées d'une autonomie renforcée, de ressources et de responsabilités précises, les villes s'affirment comme des acteurs de premier plan du développement territorial. 
25 En parallèle, le gouvernement amorce une restructuration de la hiérarchie administrative entre les territoires. Trois politiques sont mises en œuvre dans les années 1980 (Ma, 2005) :

1. la municipalité gouverne le district (shi guan xian ou shi dai xian);

2. la conversion de districts en municipalités (xian gai shi ou zheng xian gai shi) ;

3. l'annexion de districts suburbains par les municipalités (che xian she qu).

Ces politiques ont pour objectif d'asseoir le pouvoir des municipalités au sein de l'administration territoriale, et ainsi de renforcer le pouvoir des villes sur les périphéries rurales et de faciliter leur développement en leur donnant la possibilité d'administrer de vastes territoires ruraux.

En augmentant les pouvoirs des gouvernements urbains et en leur donnant la prévalence sur les gouvernements ruraux, le gouvernement central a conforté l'emprise de la ville tant au niveau régional que local. Ces changements ont modifié la forme de l'aménagement du territoire. Les villes-centres en sont devenues les maîtres d'œuvre, non seulement à l'échelle de leur périmètre urbain mais aussi à l'échelle de leur territoire administratif. La ville gère donc la construction des infrastructures de réseau (route, électricité, eau potable, assainissement, déchets) sur des territoires souvent de plusieurs milliers de $\mathrm{km}^{2}$. Les services urbains se diffusent vers les bourgs et les périphéries rurales à partir de la ville-centre, qui seule dispose des institutions capables de gérer ces investissements. C'est également elle qui a la charge du plan directeur de développement de la municipalité. La forme de l'aménagement des territoires se retrouve par conséquent centrée sur cette entité, devenue un véritable pôle structurant.

Ainsi toutes les villes centres-administratifs des municipalités et des districts ont-elles de nouveaux pouvoirs élargis à de vastes territoires, et ce quelle que soit leur taille. Cette évolution de l'administration territoriale a impulsé l'essor de la petite ville et a dessiné la forme du développement territorial: les petites villes se démarquent des échelons administratifs supérieurs et prennent une certaine autonomie. Elles ont alors initié une réorganisation spatiale du territoire, caractérisée par un polycentrisme, qui s'exprime différemment selon les grands ensembles de la province.

\section{De nouveaux centres qui recomposent les territoires}

\section{L'évolution d'un desakota dans la région deltaïque}




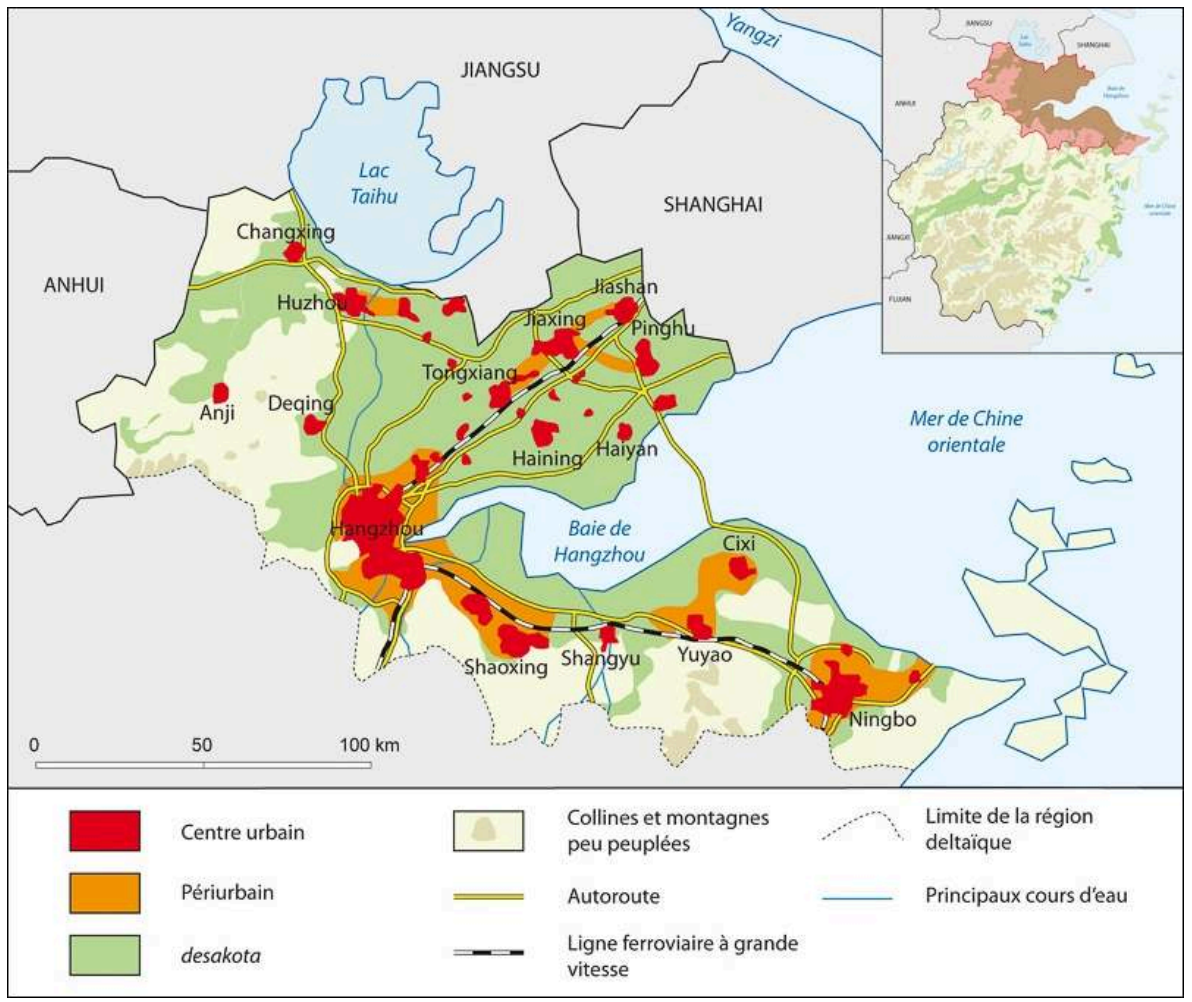

Source : Google Earth, 2012

Dans le cas de la région deltaïque du nord du Zhejiang, on trouve un exemple de l'évolution d'un desakota asiatique dans un environnement où la croissance est très forte et où la structure administrative territoriale soutient le développement de centres secondaires indépendants. Depuis trente ans, l'industrialisation rurale y est le mode de développement dominant. Le desakota initial se construit autour d'un axe de communication qui va de Shanghai à Hangzhou puis de Hangzhou à Ningbo. Cet axe concentre les infrastructures de transport à grande vitesse, ainsi que de nombreux centres urbains : Jiashan, Pinghu, Jiaxing, Tongxiang et Haining entre Shanghai et Hangzhou ; Shaoxing, Shangyu et Yuyao entre Hangzhou et Ningbo. La densité y est telle qu'il y a une petite ville en moyenne tous les $34 \mathrm{~km}$ entre Shanghai et Hangzhou et tous les $42 \mathrm{~km}$ entre Hangzhou et Ningbo (cf. illustration 7) ${ }^{21}$.

Depuis 2008, le pont de la baie de Hangzhou a créé une liaison transversale entre Yuyao et Jiashan en passant par Cixi et Pinghu. Le renforcement d'autres liaisons transversales est en cours : tout d'abord le long du Grand Canal entre Hangzhou et Huzhou en passant par Deqing, puis entre Shanghai et Huzhou (via Jiaxing), liaison qui se prolonge vers Hefei dans la province de l'Anhui. Le desakota change de visage, il se densifie et les corridors de développement s'y multiplient. Au sein de cet ensemble, la hiérarchie entre les centres s'est complexifiée avec le développement des petites villes. Deux villes moyennes se sont imposées, Jiaxing et Huzhou, qui structurent le nord de la plaine deltaïque. Autour d'elles et des deux grandes villes, Hangzhou et Ningbo, plusieurs petites villes s'affirment et se transforment. Toutes sont des centres industriels qui évoluent en centres tertiaires. Cette mutation a en parallèle entraîné le développement de nouveaux bourgs ruraux qui ont profité du glissement des activités industrielles. 
31 Le développement à l'intérieur de la plaine deltaïque est ainsi polycentrique, et se démarque dans sa forme de développement d'un desakota par la multiplication et la hiérarchisation des centres qui structurent chacun à leur niveau le territoire. Les corridors de développement, décrits par McGee le long des axes de communication, s'étendent désormais à l'ensemble de l'espace deltaïque tant le maillage s'est densifié, et ils perdent leur caractère discriminant. Le territoire ne s'organise plus uniquement autour des grandes villes : au-delà des périphéries de Shanghai, les centres secondaires ont leurs propres logiques de développement. Le territoire se conçoit tel un millefeuille où à chaque couche correspond une catégorie de ville :

1. la couche des grandes villes rassemble les infrastructures internationales telles que les aéroports ou les ports internationaux, le secteur des nouvelles technologies, le secteur financier et les universités de haut niveau ;

2. la couche des villes moyennes regroupe les infrastructures de transport de niveau régional, tels qu'autoroutes et lignes ferroviaires à grande vitesse, et les équipements de loisirs et éducatifs au rayonnement régional ;

3. la couche des petites villes est connectée au réseau d'infrastructures à grande vitesse et elle évolue vers une économie tertiaire. Chacune d'entre elles est le centre d'une redistribution spatiale des réseaux, des activités et des populations.

La logique de projets, qui dessine la forme du développement territorial, est pilotée désormais par l'échelon des petites villes. Elle est à l'origine de l'évolution polycentrique du desakota. La poussée des centres secondaires a été telle qu'ils impulsent leur propre forme du développement au sein de leur espace administratif. Ils s'émancipent aussi bien de la métropole, Shanghai, que des grandes villes de la plaine deltaïque que sont Hangzhou et Ningbo. Le desakota a donc bien évolué au cours des deux dernières décennies : le territoire n'est plus structuré seulement par des grandes villes et des corridors les reliant où se diffusent les activités urbaines, mais par une multitude de centres hiérarchisés, qui, chacun à leur niveau, organisent la répartition des activités et des populations.

\section{Un chapelet de centres urbains sur le littoral}

La configuration spatiale de la côte, coincée entre mer et montagne, a dessiné une armature urbaine composée d'une succession de petites et de deux grandes villes, Wenzhou et Taizhou, que l'on trouve les unes à la suite des autres selon l'axe naturel nord-sud. La distance moyenne entre deux villes est de $42 \mathrm{~km}^{22}$, soit une distance ici encore relativement faible (cf. illustration 8).

Leur taille dépend étroitement de la topographie, ce qui explique qu'une seule ville moyenne, Rui'an, s'y soit développée au cours des dix dernières années. Les deux grandes villes occupent déjà les plaines les plus importantes, et Rui'an s'est développée dans le prolongement de Wenzhou au sein de la même plaine côtière. Les autres villes, fortement contraintes par la topographie, sont dans l'impossibilité physique de s'étendre. De facto, le développement polycentrique est arrivé à son terme sur le littoral, les possibilités de création de nouvelles villes ayant été épuisées. Ainsi le littoral présente-t-il un chapelet éclectique de villes qui aujourd'hui atteignent déjà leur taille critique. 
Illustration 8 - Un chapelet de centres urbain sur le littoral

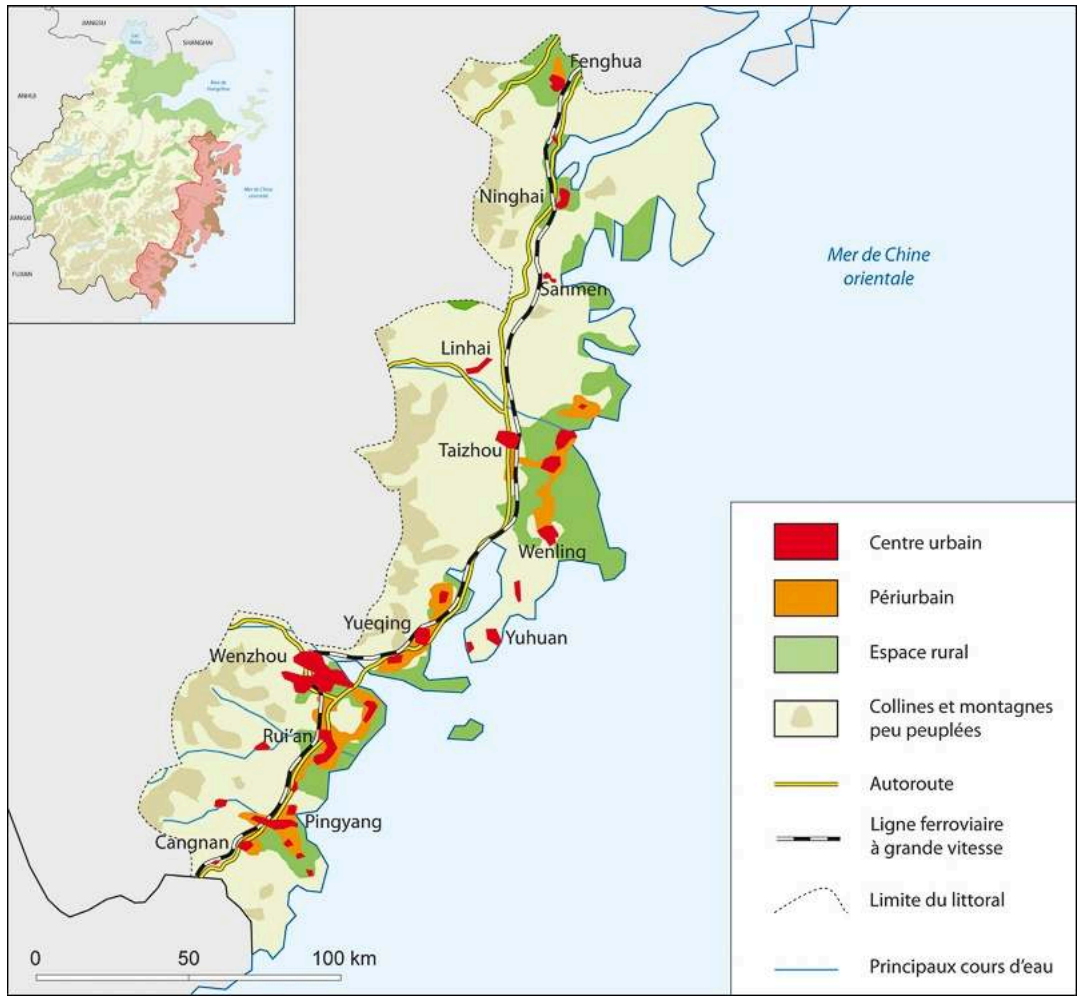

Source : Google Earth, 2012.

L'arrivée du réseau autoroutier puis du train à grande vitesse connecte désormais ces villes côtières au reste de la province : aujourd'hui intégrées au réseau urbain régional, qui se prolonge vers le nord à l'ensemble du delta du Yangzi et vers le sud à la province du Fujian, elles ne sont plus seulement tournées vers la mer mais regardent aussi vers les terres intérieures et la région deltaïque. Ce réseau permet aux petites villes saturées du littoral de déplacer une partie de leurs activités industrielles vers l'intérieur des terres et de poursuivre leur transition vers le secteur tertiaire.

\section{La formation d'un réseau urbain dans les terres de l'intérieur}

Dans cet ensemble, la distance moyenne entre les villes est plus importante que dans le reste de la province : $58 \mathrm{~km}$ ( $c f$. illustration 9$)^{23}$. Deux nouvelles villes moyennes, Yiwu et Jinhua, structurent le bassin du Jinqu. L'armature urbaine se compose ensuite de petites villes situées le long des deux axes Yiwu-Jinhua-Quzhou et Jinhua-LishuiWenzhou, ainsi que d'autres petites villes dispersées dans les montagnes périphériques. Suivant que l'on se trouve dans le bassin du Jinqu ou dans les montagnes, les modes de développement diffèrent. Dans le bassin, les mêmes dynamiques que dans la région deltaïque sont à l'œuvre, avec un développement polycentrique structuré par les petites villes. Dans les montagnes, la topographie contraint la forme du développement qui se concentre sur les centres existants, à l'image de la situation sur le littoral. C'est le cas par exemple à Lishui ou à Jiande.

À l'inverse de la région deltaïque ou du littoral, où les réseaux de transport n'ont pas de rôle discriminant dès lors qu'ils desservent tous les centres urbains, ils ont un rôle 
déterminant dans les terres intérieures. C'est le cas notamment de la nouvelle ligne ferroviaire à grande vitesse qui longe le bassin du Jinqu et relie Hangzhou à Nanchang dans la province voisine du Jiangxi, en passant par Zhuji, Yiwu, Jinhua, Longyou, Quzhou et Jiangshan. L'autre axe structurant de cet ensemble est l'autoroute reliant Jinhua à Wenzhou via Lishui. Ces deux axes sont des vecteurs d'intégration des terres intérieures au réseau urbain régional. Le mode de développement des terres intérieures intègre le déplacement des activités industrielles depuis la région deltaïque et le littoral pour des raisons de coût et d'espace, accompagné d'un développement du secteur tertiaire des centres urbains. Les réseaux de transport et les disponibilités foncières participent à la sélection des petites villes qui accueilleront les nouvelles industries.

Illustration 9 - La formation d'un réseau urbain dans les terres de l'intérieur

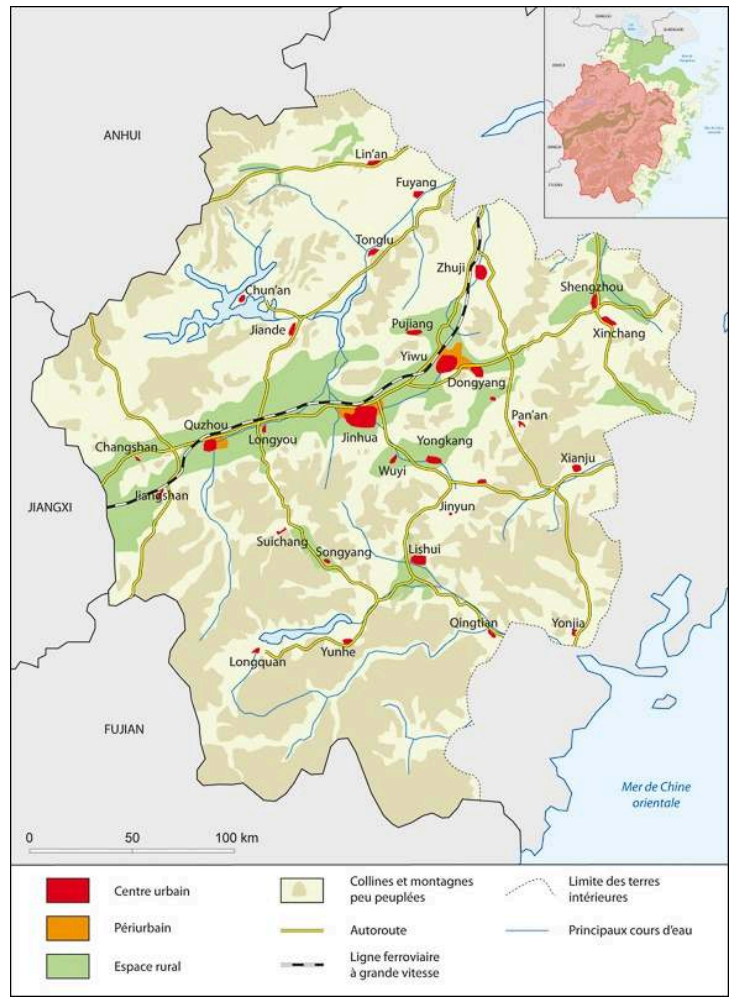

Source : Google Earth, 2012.

\section{Conclusion}

L'émergence et la croissance des petites villes et leur évolution ont multiplié les centralités au sein du desakota dans la région deltaïque, elles ont confirmé le développement des centres existants sur le littoral désormais connecté au reste de la province, et elles ont impulsé le développement des terres intérieures en constituant un réseau urbain régional dense qui se prolonge jusque dans les provinces voisines. Dans chaque ensemble de la province, les petites villes sont à l'origine d'un développement polycentrique, façonné par un système administratif territorial singulier qui a contribué à les émanciper et à leur donner les moyens de leur autonomie. 
40 L'illustration 10 décrit l'armature urbaine du Zhejiang en 2010. Elle souligne un développement des petites villes à l'origine tant d'une distribution plus équilibrée de la population entre grands centres et centres secondaires que d'une meilleure répartition de la production de la richesse sur l'ensemble du territoire. Les tendances actuelles dans la province du Zhejiang vont donc vers une densification et une hiérarchisation de l'armature urbaine. La forme du système administratif territorial a multiplié les centres secondaires. Cependant, n'autorisant le développement effectif que d'un unique centre urbain par municipalité et par district, ce développement polycentrique touche à son terme, et l'on entre aujourd'hui dans une phase de confirmation de la croissance de ces centres secondaires.

41 Le Zhejiang met en évidence les résultats d'une politique d'aménagement du territoire volontariste dans le contexte d'une économie dynamique en transition. Les grands pôles urbains évoluent vers le secteur tertiaire, le secteur financier ou encore celui des nouvelles technologies. Cette transition entraîne, pour des raisons de coûts ou d'espace, un glissement d'une partie des facteurs de production tel que ceux des secteurs du textile, du mobilier, de l'électroménager ou de l'automobile. Cependant, plutôt que de se concentrer dans les périphéries des grands centres existants, ils migrent vers de nouveaux centres secondaires. La politique du gouvernement, visant à donner aux petites villes les moyens de se développer par la décentralisation, un encadrement administratif du territoire à leur avantage mais aussi de lourds investissements dans les réseaux de transport et les infrastructures sanitaires, contribue à l'équilibre de l'armature urbaine. Cela se traduit par une meilleure répartition des populations et des activités économiques sur le territoire et par une diminution de la pression démographique, économique et urbaine sur les plus grands centres. Ce développement polycentrique modifie les rayons d'influence des grandes villes, notamment à travers le tracé des réseaux de communication. Certes le développement régional chinois est-il marqué par la formation de grands espaces métropolitains, mais aussi par l'apparition de centres secondaires qui participent à la diffusion progressive de la croissance sur le territoire. Tous n'en bénéficieront pas de façon soutenue et une inévitable sélection va s'opérer dans des territoires encore en marge du développement. Mais incontestablement, dans cette nouvelle hiérarchie urbaine, les petites villes en sont les acteurs préférentiels. 
Illustration 10 - L'armature urbaine dans la province du Zhejiang en 2010

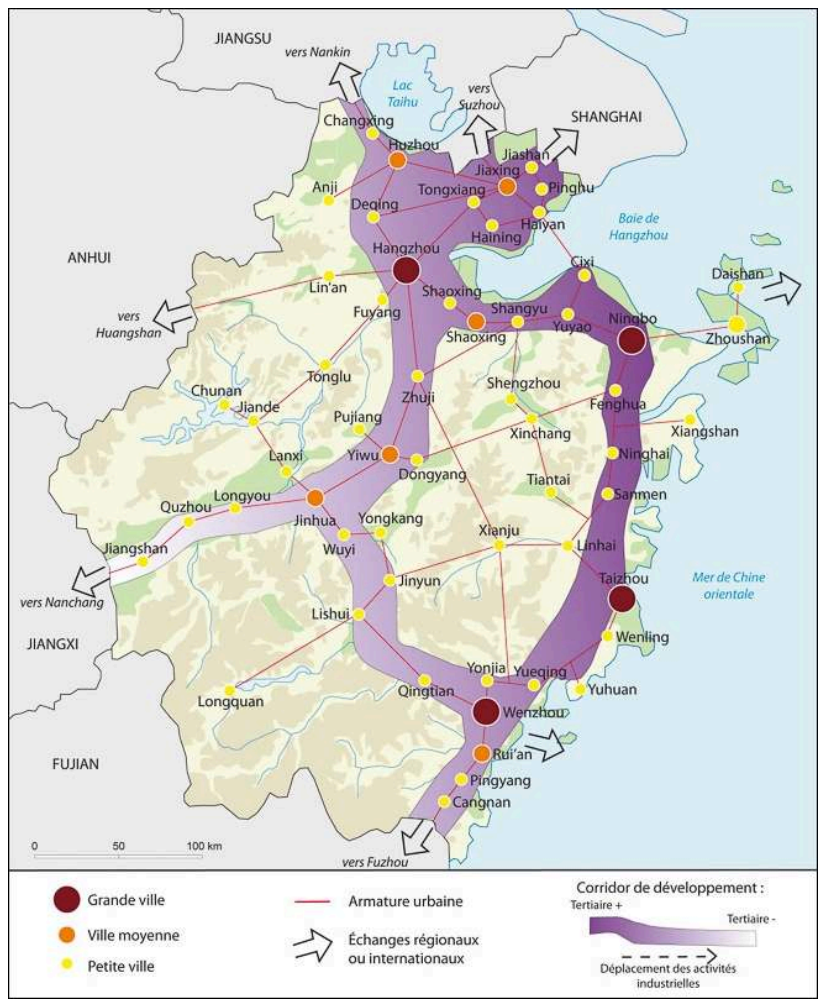

\section{BIBLIOGRAPHIE}

Banque asiatique de développement, 2005. Town-Based Urbanization for Balanced Development of Urban System. Manille, $44 \mathrm{p}$.

Banque mondiale, 2008. Small towns in China: A Strategic Approach to Rationalizing the Urban Hierarchy. Washington, $30 \mathrm{p}$.

Banque mondiale, 2012. China Small and Medium Towns Overview. Washington, 56 p.

Cabestan J.-P., 1992. L'administration chinoise après Mao. Les réformes de l'ère de Deng Xiaoping et leurs limites. Paris, CNRS, $545 \mathrm{p}$.

Chan K. W., Xu X., 1985. Urban population growth in China since 1949: reconstructing a Baseline. The China Quarterly, $\mathrm{n}^{\circ}$ 104, p. 583-613.

Chan K. W., 1994a. Cities with invisible walls: reinterpreting urbanization in post-1949 China. Oxford, Oxford University Press, 193 p.

Chan K. W., 1994b. Urbanization and rural-urban migration in China since 1982: a new baseline. Modern China, vol. 20, n 3, juillet, p. 243-281.

Gentelle P., 1992. Chine : l'irrésistible urbanisation. Problèmes économiques et sociaux, $\mathrm{n}^{\circ} 682,61 \mathrm{p}$.

Kamal-Chaoui L., Leman E., Rufei Z., 2009. Urban Trends and Policy in China. Paris, OCDE, 70 p. 
Lu D, 2010. Regional development research in China: a roadmap to 2050. Pékin, Science Press, 192 p.

Ma L., 2002. Urban Transformation in China, 1949-2000: a review and research agenda. Environment and Planning A, 34(9), p. 1545-1569.

Ma L., 2005. Urban administrative restructuring, changing scale relations and local economic development in China. Political Geography, n² 25, p. 487-489.

McGee T., 1991. The emergence of desakota regions in Asia: expanding a hypothesis. In Ginsburg N. et al. (dir.), The Extended Metropolis. Settlement Transition in Asia, Honolulu, University of Hawai Press, p. 3-25.

McKinsey Global Institute, 2009. Preparing for China's urban billion. New York, 539 p.

Sanjuan T., 1996. Qu'est-ce qu'une population urbaine en Chine ? L'exemple du delta de la rivière des Perles (province du Guangdong). Géographie et cultures, n 19, p. 33-49.

Sanjuan T., 1997. À l'ombre de Hong Kong, le delta de la Rivière des Perles. Paris, L'Harmattan, 313 p.

Sanjuan T., 2000. La Chine. Territoire et société. Paris, Hachette, 189 p.

Sanjuan T., 2007. Approcher les dynamiques régionales en Chine. Hérodote, n 125, p. 157-185.

Sanjuan T. (dir.), 2007. Dictionnaire de la Chine contemporaine. Paris, Armand Colin, 268 p.

Skinner G. W., 1977. The City in Late Imperial China. Stanford, Stanford University Press, 820 p.

Wei Y., Ye X., 2005. Geospatial Analysis of Regional Development in China: The Case of Zhejiang Province and the Wenzhou Model. Eurasian Geography and Economics, n 5, p. 342-361.

Zhang M., 2009. Zhejiang Chengshihua 30 nian [30 ans d'urbanisation dans le Zhejiang], Hangzhou, Zhejiang Renmin Chubanshe, 306 p.

Zhejiang sheng dituji [Atlas du Zhejiang], 2008, Pékin, Zhonguo ditu chubanshe, 326 p.

Zhejiang sheng 1990 nian renkou pucha ziliao [Résultats du recensement de la population de la province du Zhejiang en 1990], 1992, Pékin, Zhongguo tongji chubanshe, 4 vol., 1768 p.

Zhejiang sheng 2000 nian renkou pucha ziliao [Résultats du recensement de la population de la province du Zhejiang en 2000], 2002, Pékin, Zhongguo tongji chubanshe, 4 vol., 1854 p.

Zhejiang sheng 2010 nian renkou pucha ziliao [Résultats du recensement de la population de la province du Zhejiang en 2010], 2012, Pékin, Zhongguo tongji chubanshe, 4 vol., 1832 p.

Zhongguo tongji nianjian [Annuaire Statistique de la Chine], 2011, Pékin, Zhongguo tongji chubanshe, $1057 \mathrm{p}$.

Zhejiang tongji nianjian [Annuaire Statistique du Zhejiang], 2000, Pékin, Zhongguo tongji chubanshe, $664 \mathrm{p}$.

Zhejiang tongji nianjian [Annuaire Statistique du Zhejiang], 2005, Pékin, Zhongguo tongji chubanshe, $752 \mathrm{p}$.

Zhejiang tongji nianjian [Annuaire Statistique du Zhejiang], 2011, Pékin, Zhongguo tongji chubanshe, $672 \mathrm{p}$.

\section{NOTES}

1. Zhongguo tongji nianjian [Annuaire statistique de la Chine], 2011, p. 4-5.

2. Zhongguo tongji nianjian, 2011, p. 4-5. 
3. Pour les années à venir, différentes sources s'accordent sur des chiffres similaires, liés à une encore très importante surpopulation rurale. On retrouve les prévisions de Han Jun, directeur du département de la recherche sur l'économie rurale au Conseil des affaires d'État, dont les études estiment que la population rurale, aujourd'hui environ 700 millions de personnes, sera de 400 millions d'ici trente ans: http://french.peopledaily.com.cn/Economie/6902379.html, 25 février 2010. UN Habitat estime de son côté qu'il y aura 400 millions de nouveaux urbains en Chine dans les deux à trois prochaines décennies (données statistiques en ligne de UN Habitat: http://www.unhabitat.org/stats/Default.aspx).

4. Georges William Skinner note alors l'expression d'un développement régional différencié qui, à l'échelle du pays, relève de l'intensité des échanges, de la ramification des réseaux et de la densité de la population (Skinner, 1977, p. 3-32).

5. En particulier la Banque mondiale et la Banque asiatique de développement.

6. Les systèmes urbains régionaux y sont identifiés suivant les critères suivants: densité de population, volume du trafic journalier moyen sur le réseau autoroutier, densité du réseau autoroutier existant et planifié, densité du réseau ferroviaire existant et planifié.

7. McKinsey Global Institute classe les villes suivant les catégories suivantes : les megacities ont plus de 10 millions d'habitants; les grandes villes entre 5 et 10 millions; les villes moyennes entre 1,5 et 5 millions; les petites villes entre 500000 et 1,5 million; et les gros bourgs moins de 500000.

8. Zhongguo tongji nianjian, 2011, p. 57 pour le PIB, p. 95 pour la population.

9. La plaine deltaïque recouvre les préfectures de Hangzhou, Ningbo, Jiaxing, Huzhou, Shaoxing et Zhoushan (Zhejiang tongji nianjian [Annuaire Statistique du Zhejiang], 2011, p. 532).

10. Le littoral recouvre les préfectures de Wenzhou et de Taizhou (Zhejiang tongji nianjian, 2011, p. 532).

11. Le bassin de la rivière Qiantang recouvre les préfectures de Jinhua, Quzhou et Lishui (Zhejiang tongji nianjian, 2011, p. 532).

12. Zhejiang sheng 2010 nian renkou pucha ziliao [Résultats du recensement de la population de la province du Zhejiang en 2010], 2012, vol. 1, p. 2-7.

13. Zhuo J., 2007. Urbanisme. In Sanjuan T. (dir.), Dictionnaire de la Chine contemporaine, p. 264.

14. Dans la province du Zhejiang, la taille moyenne des municipalités est de $1509 \mathrm{~km}^{2}$ (Zhejiang tongji nianjian [Annuaire Statistique du Zhejiang], 2011, p. 531).

15. Si les trois recensements répertorient la population en fonction du hukou d'origine, qui ne recoupe pas précisément les espaces urbains et ruraux mais permet de dégager les tendances en cours (Sanjuan, 1996, p. 33-49), les recensements de 2000 et 2010 y ajoutent la répartition de la population en fonction du type de lieu de vie : ville-centre, bourg ou village.

16. Le hukou est un système administratif d'enregistrement des populations instauré en 1958, dont l'objectif est, du moins au départ, de contrôler strictement les migrations. Les citoyens chinois se voient attribuer un statut résidentiel et économique à la naissance, principalement héréditaire. Deux indications principales y sont portées : le nom de la localité où le hukou a été enregistré et le statut du hukou possédé, agricole ou non agricole. Ces deux critères définissent relation de l'individu avec l'État. (Thireau I., 2007. Enregistrement de la résidence. In Sanjuan, 2007, p. 89-91).

17. Le recensement de 1990, basé sur les chiffres du hukou, dessine une armature urbaine composée de trois principaux centres (Hangzhou, de loin le plus peuplé avec une population non agricole de 1133556 habitants, Ningbo avec 557680 habitants et Wenzhou avec 417685 habitants), et d'un réseau de treize villes secondaires, dont la population non agricole allait de 100000 à 200000 habitants.

18. Zhejiang sheng 2000 nian renkou pucha ziliao [Résultats du recensement de la population de la province du Zhejiang en 2000], 2002, vol. 1, p. 2-7. 
19. En dehors des secteurs ou des intérêts stratégiques, ils décident dès lors de l'affectation des investissements aussi bien nationaux qu'étrangers, et plus généralement initient les projets de développement; ils ont la maîtrise du foncier et par là même, de la destination des terrains : la compétence en matière d'urbanisme est, sauf certaines exceptions, décentralisée au niveau des gouvernements municipaux; ils gèrent les investissements publics et les services urbains ; un système fiscal forfaitaire est adopté pour les villes, le gouvernement central ne prélevant qu'une somme forfaitaire sur les recettes fiscales locales, le solde restant à la disposition des gouvernements municipaux ; le système actuel de crédit-bail à long terme d'utilisation du foncier est mis en place, et ouvre de nouvelles sources de financement aux gouvernements locaux. (Ma, 2002 ; Cabestan, 1992 p. 247-256).

20. Orientation du $\mathrm{VI}^{\mathrm{e}}$ plan quinquennal (1981-1985).

21. https://maps.google.com/ (site Internet consulté en décembre 2012).

22. https://maps.google.com/ (site Internet consulté en décembre 2012).

23. https://maps.google.com/ (site Internet consulté en décembre 2012).

\section{RÉSUMÉS}

Depuis l'ouverture économique de la Chine en 1978, ce sont plus de 15 millions de nouveaux citadins que les villes accueillent chaque année. La progression du taux d'urbanisation est exceptionnelle : de 17,9\% en 1978 à 49,9 \% en 2010. Quels sont les impacts de l'ampleur de cette urbanisation sur l'armature urbaine? Cet article s'intéresse au cas de la province du Zhejiang, située au sud de Shanghai, dont le taux d'urbanisation a augmenté sur cette période de 13,7 à $61,6 \%$. Entre la formation de régions métropolitaines concentrant population et activités économiques et caractérisées par de fortes densités humaines, et le scénario soutenu par le gouvernement de leur répartition plus équilibrée sur le territoire, ce texte analyse l'évolution de la distribution des villes et la forme du développement régional dans cette province riche du littoral.

In China, since the economic opening in 1978, more than 15 million people move to cities every year. The urban rate has grown from $17.9 \%$ in 1978 to $49.9 \%$ in 2010: the unprecedented size and rapidity of Chinese urbanization have reformed the urban framework through the country. This article focuses on Zhejiang province, located at the south of Shanghai, which urban rate has increased from 13.7 to $61.6 \%$ during the same period. It analyses the urban framework changes, between on the one hand the development of clusters and metropolitan areas that concentrate population and economic activities, and on the other hand the government policy that aims to balance the distribution of cities.

\section{INDEX}

Mots-clés : armature urbaine, développement régional, desakota, région urbaine, centralité, polycentrisme, administration territoriale

Keywords : Urban framework, regional development, desakota, cluster, centrality, polycentric development

Thèmes : Sur le Champ - Sur le Terrain 


\section{AUTEUR}

\section{STÉPHANE MILHAUD}

Stéphane Milhaud, stephanem@groupehuit.com ingénieur-urbaniste de la société Groupe Huit, est docteur en géographie (Université Paris 1 Panthéon-Sorbonne). 